\title{
A prospective randomised, controlled clinical trial comparing medial and lateral entry pinning with lateral entry pinning for percutaneous fixation of displaced extension type supracondylar fractures of the humerus in children
}

Abhijan Maity ${ }^{1,3^{*}}$, Debasish Saha ${ }^{2}$ and Debasis Sinha Roy ${ }^{1}$

\begin{abstract}
Objective: To compare the efficacy of medial and lateral entry pinning with lateral entry pinning for percutaneous fixation of displaced (Gartland type II and type III) extension type supracondylar fractures of the humerus in children.

Methods: The study was a single center, prospective, randomized controlled clinical trial. Between October 2007 and September 2010, 160 patients who satisfy the inclusion and exclusion criterias were enrolled in the study, with 80 patients in each group. All the percutaneous pinning was done according to a uniform standardized technique. The patients were re-evaluated as outpatients at three weeks, six weeks and three months after the surgery. At three months follow-up visit, following informations were recorded as outcome measures: (i) Carrying angle (deg) (ii) passive range of elbow motion (deg) (iii) Flynn's criteria for grading, based on the loss of carrying angle and loss of total range of elbow motion. (iv) Baumann angle (deg) (v) Change in Baumann angle (deg) between the Intraoperative radiographs after the surgery and radiographs at three months follow-up visit (vi) loss of reduction grading, based on the change in the Baumann angle.
\end{abstract}

Results: There were no significant differences between the two groups with regard to base-line characteristics, withdrawals and complication rate. At three months follow-up visit, patients were evaluated by recording the various outcome measures. There were no significant differences between the two groups with regard to the various outcome measures such as carrying angle, passive range of elbow motion, Flynn grading, Baumann angle, change in the Baumann angle and loss of reduction grading.

Conclusions: If a uniform standardized operative technique is followed in each method, then the result of both the percutaneous fixation methods will be same in terms of safety and efficacy.

Keywords: Supracondylar fractures, Humerus, Children, Percutaneous fixation

\section{Introduction}

Supracondylar fractures of the humerus are the most common fractures about the elbow in children [1,2]. According to Boyd and Altenberg [3], these fractures account for $65.4 \%$ of upper extremity fractures in children. Gartland [4] proposed a classification for these

\footnotetext{
* Correspondence: abhijan.maity@gmail.com

'Department of Orthopaedics, Burdwan Medical College, Burdwan, West Bengal, India

Full list of author information is available at the end of the article
}

fractures: type I, Undisplaced; type II, displaced with the posterior cortex intact; and type III, completely displaced with no cortical contact. Supracondylar fractures may be associated with a number of complications such as neurovascular injuries, malunion, compartment syndrome, iatrogenic neurovascular injury and elbow stiffness $[1,2,5]$. Cubitus varus due to malunion is the most common angular deformity and the incidence varies from 5\% according to Flynn et al. [6], to $21 \%$ according to Arino et al. [7]. Incidence of iatrogenic ulnar nerve 
injuries after percutaneous fixation with medial and lateral entry pinning was about $15 \%$ according to Chai [8].

The recommended method of treatment for displaced (Gartland [4] type II and type III) extension type Supracondylar fractures of the humerus in children is closed reduction and percutaneous pin fixation. But, the optimal method of percutaneous pin fixation varies among authors. Swenson [9], Casiano [10] and Flynn et al. [6] used two crossed pins, one introduced medially and one laterally. Arino et al. [7] used two lateral pins.

Though crossed medial-lateral pin fixation provides increased biomechanical stability, but simultaneously it carries the risk of iatrogenic ulnar nerve injury from placement of the medial pin [11-13]. Conversely, the twolateral pin fixation avoids the danger of iatrogenic ulnar nerve injury, but it provides less biomechanical stability [14-18].

The aim of our present study was to compare the efficacy of medial and lateral entry pinning with lateral entry pinning for percutaneous fixation of displaced (Gartland [4] type II and type III) extension type supracondylar fractures of the humerus in children.

\section{Materials and methods Trial designs}

The study was a single center, prospective, randomized controlled clinical trial, conducted in Department of Orthopaedics and Traumatology of our institution from October 2007 to September 2010. The protocol was approved by the ethics committee of our institution. Randomization was done after we had taken written informed consent from the study participants and obtained base line information. The random assignment scheme was created from a table of random numbers. Opaque prenumbered sealed envelopes containing random assignments were maintained by the hospital pharmacist.

\section{Patients}

All the children attending in the Accident and Emergency Department or, in the Outpatient Department of Orthopaedics and Traumatology in our institution between October 2007 and September 2010 with supracondylar fractures of the humerus were enrolled in the present study if they had the following inclusion criterias: (i) age between two and twelve years (ii) Unilateral fracture (iii) Extension type (iv) Displaced Gartland [4] type II and type III (v) presenting within seventy two hours after the injury (vi) no other associated injury in the same limb (vii) no previous fracture in the same limb.

Patients were excluded if they fulfill the following exclusion criterias: (i) age less than two years or, greater than twelve years (ii) Bilateral fracture (iii) Flexion type (iv) Undisplaced Gartland [4] type I (v) presenting more than seventy two hours after the injury (vi) associated injury in the same limb (vii) Previous fracture in the same limb (viii) open fracture (ix) unsatisfactory closed reduction requiring open reduction $(\mathrm{x})$ floating elbow (xi) failure to perform a preoperative neurovascular examination (xii) associated neurovascular injury requiring surgical exploration

\section{Treatment}

Surgery was done under general anesthesia. All the patients were positioned supine on a fracture table and closed reduction were performed under the fluoroscopic guidance. If the closed reduction was satisfactory, then percutaneous fixation with either crossed medial-lateral pin or, two-lateral pin was done according to the random assignment scheme.

All the crossed medial-lateral pinning was done according to the mini-open technique described by Green et al. [19]. All the lateral entry pinning was done according to the technique described by Aroson and Prager [20].

Intraoperative radiographs obtained after pin placement was accepted in all cases. Loss of reduction were observed in postoperative radiographs of nine children who underwent crossed medial-lateral pin fixation as shown in Figure 1 and in postoperative radiographs of eight children who underwent two lateral pin fixation as shown in Figure 2.

Pin-tract infection was defined as documentation of purulent or, seropurulent discharge/erythema around the pin-site, with or without bacteriological evidence of infection.

Betadine soaked gauzes were placed around each pin site and a kling flexible dressing bandage was then used as an occlusive dressing around all the pin sites. The dressing was left in place for 48 hours. Thereafter, each pin site was cleaned with normal saline for removal of crust. In the presence of exudates, a light gauze dressing was applied. In the absence of exudates, the pin sites were left uncovered. A single preoperative parenteral dose of Cefuroxime was given at the time of induction, which was continued post-operatively for 72 hours. The patient was discharged with advice of taking oral antibiotics only if a pin site infection was developed. The signs and symptoms of a pin site infection were clearly explained to the parents (pain, erythema, tenderness, discharge). Parents were instructed to clean the pin sites daily with cotton swabs soaked in normal saline to remove all crusts.

\section{Follow-up and outcome measure}

The patients were re-evaluated as outpatients at three weeks, six weeks and three months after the surgery. Follow-up assessment of each patient was done by the same doctor throughout the trial. Both the surgeons and the patients were not blinded of the treatment received 


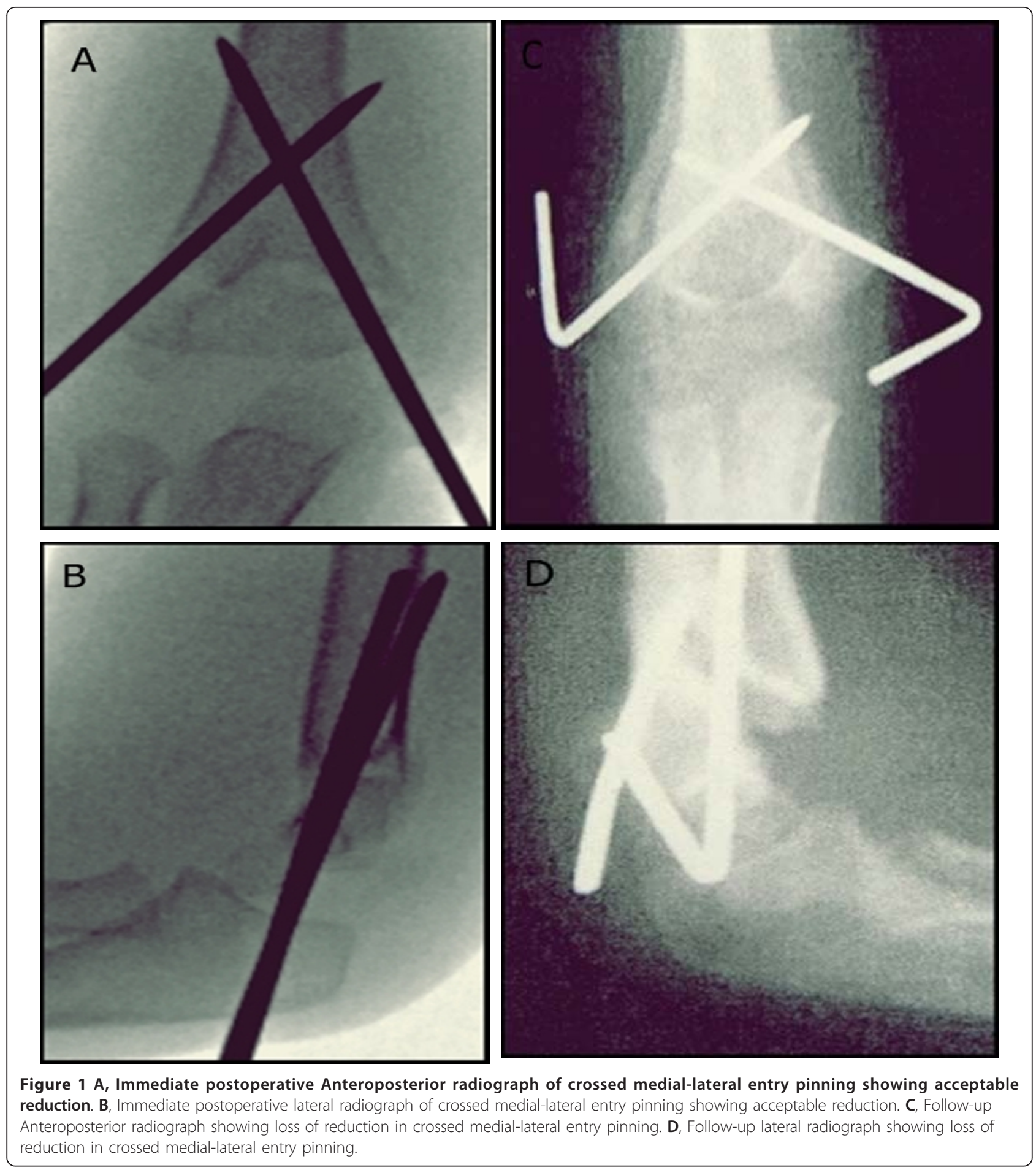

throughout the trial. Plaster cast and pins were removed at three weeks. At three months follow-up visit, following informations were recorded as outcome measures: (i) Carrying angle (deg) (ii) passive range of elbow motion (deg) (iii) Flynn's criteria for grading [6], based on the loss of carrying angle and loss of total range of elbow motion (Table
1). (iv) Baumann angle (deg), calculated on the skiagram of anteroposterior view of elbow with the method described by Williamson et al. [21]. (v) Change in Baumann angle (deg) between the Intraoperative radiographs after the surgery and radiographs at three months follow-up visit (vi) loss of reduction grading, based on the change in the 


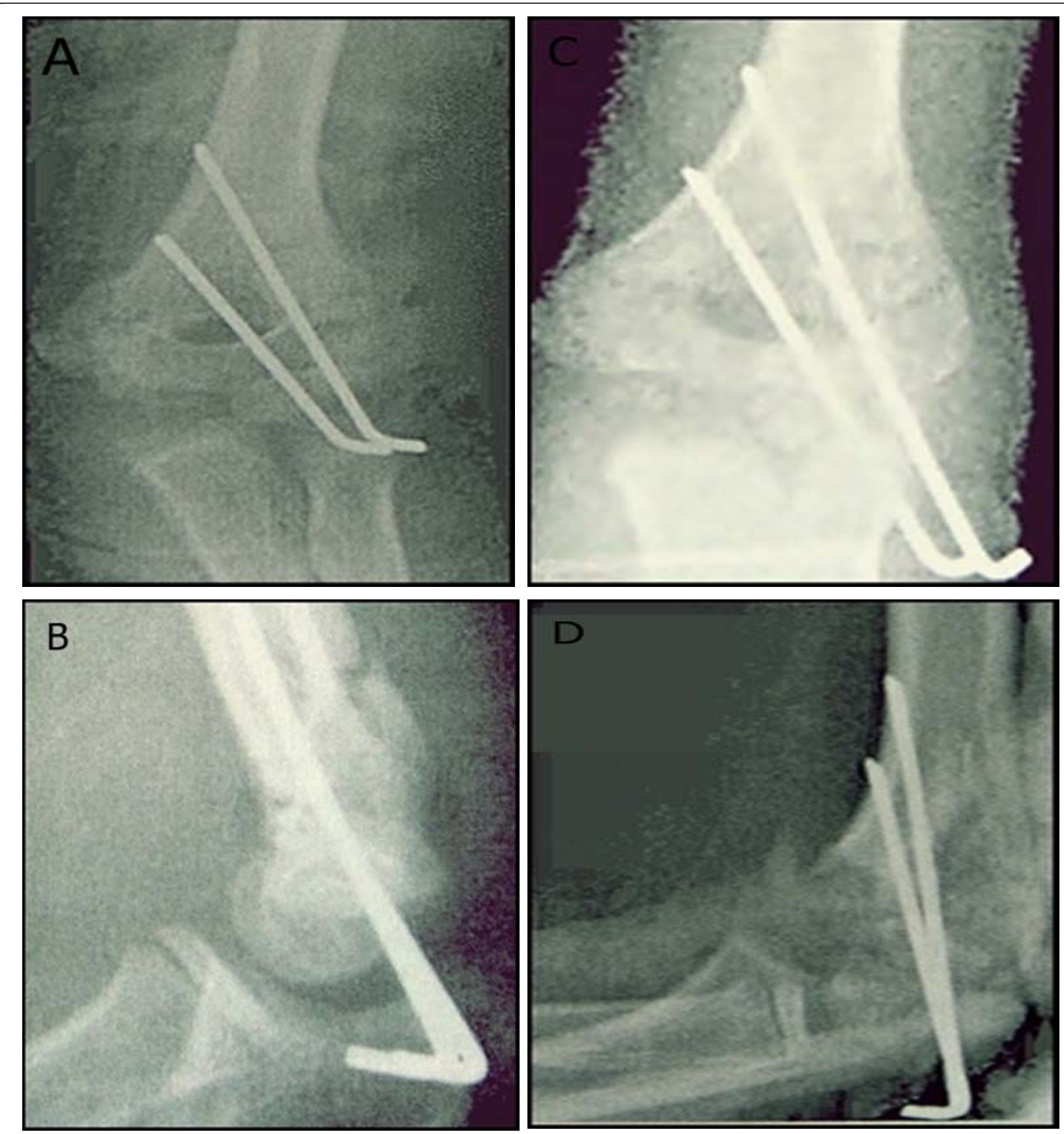

Figure 2 A, Immediate postoperative Anteroposterior radiograph of two-lateral entry pinning showing acceptable reduction. B Immediate postoperative lateral radiograph of two-lateral entry pinning showing acceptable reduction. C, Follow-up Anteroposterior radiograph showing loss of reduction in two-lateral entry pinning. D, Follow-up lateral radiographs showing loss of reduction in two-lateral entry pinning.

Baumann angle according to the method described by Skaggs et al. [22] (Table 2).

\section{Source of funding}

There was no source of external funding in support of this study.

\section{Sample size}

We selected the major loss of reduction (defined as a change in the Baumann angle of $>12^{\circ}$ between the Intraoperative radiographs and radiographs at three months follow-up visit) as the primary outcome measure at three months. We estimated that, in order to detect a $15 \%$ difference in the rates of major loss of reduction 
Table 1 Flynn's criteria for grading [6]

\begin{tabular}{llll}
\hline Result & Rating & Carrying angle loss (Degrees) & Total range of elbow motion loss (Degrees) \\
\hline Satisfactory & Excellent & $0-5$ & $0-5$ \\
\hline & Good & $5-10$ & $5-10$ \\
\hline Unsatisfactory & Fair & $10-15$ & $10-15$ \\
\hline
\end{tabular}

(with a two-sided alpha value of $5 \%$, a statistical power of $80 \%$ ) between the two groups, at least sixty patients had to be recruited in each group. We therefore planned to enroll 80 patients in each group considering for an expected maximum withdrawal rate of 25 percent.

\section{Statistical analysis}

All statistical analysis was based on an "intention to treat" principle; therefore patients who withdrew from the study, the data at the time of withdrawal were carried forward to all subsequent evaluations. The outcomes of treatment with the crossed medial-lateral pin were compared with those of treatment with the twolateral pin with the use of parametric and nonparametric analyses as appropriate for the data. The independentsample student t tests, Fisher's exact test, Pearson chisquare tests, Mann-Whitney U test were performed with use of SAS statistical package (SAS institute, Cary, North Carolina). A p value of $<0.05$ was considered to be statistically significant.

\section{Results}

\section{Study group}

Between October 2007 and September 2010, 160 patients who satisfy the inclusion and exclusion criterias were enrolled in the study, with 80 patients in the crossed medial-lateral pin entry group and 80 in the two lateral pin entry group. There were no significant differences between the two groups regarding base-line characteristics such as age, male sex, side, types of displacement, types of fracture, interval from injury to admission and interval from admission to surgery (Table 3).

\section{Withdrawals}

A total of 30 of the 160 patients did not complete the three follow-up visits. In the crossed medial-lateral pin entry group, 2 patients did not come after the surgery, 5 patients did not come after the first visit and 9 patients

Table 2 Skaggs et al. [22] criteria for grading loss of reduction

\begin{tabular}{ll}
\hline Change in Baumann angle & Loss of reduction grading \\
\hline$<6^{\circ}$ & None \\
\hline $6^{\circ}-12^{\circ}$ & Mild \\
\hline$>12^{\circ}$ & Major \\
\hline
\end{tabular}

did not come after the second visit. In the two lateral pin entry group, 2 patients did not come after the first injection, 4 patients did not come after the first visit and 8 patients did not come after the second visit. So, only 130 of the 160 patients were available at three months follow-up visit. There were no significant differences between the two groups with regard to withdrawals (Table 4). Mean number of follow-up visits in the crossed medial-lateral pin entry group $(2.7 \pm 0.7)$ was not significantly different from that in the two lateral pin entry group $(2.72 \pm 0.67)$ (Independent sample student $\mathrm{t}$ test, $\mathrm{p}=0.85$ ).

\section{Complications}

There were no significant differences between the two groups regarding neurovascular complications at the time of admission, iatrogenic ulnar nerve injury, and pin track infection (Table 5).

\section{Response to treatment}

At three months follow-up visit, patients were evaluated by recording the various outcome measures. There were no significant differences between the two groups with regard to the various outcome measures such as carrying angle, passive range of elbow motion, Flynn [6] grading, Baumann angle, change in the Baumann angle and loss of reduction grading (Table 6).

\section{Discussion}

The standard treatment for displaced (Gartland [4] type II and type III) extension type Supracondylar fractures of the humerus in children is closed reduction and percutaneous pin fixation. But, controversy persists among authors regarding optimal method of percutaneous pin fixation. Swenson [9], Casiano [10] and Flynn et al. [6] used two crossed medial-lateral pins. Arino et al. [7] used two lateral pins.

Though crossed medial-lateral pin configuration provides good biomechanical stability, but simultaneously it carries the increased risk of iatrogenic ulnar nerve injury due to placement of the medial pin [11-13]. Conversely, though the two- lateral pin configuration carries less risk of iatrogenic ulnar nerve injury, but it provides less biomechanical stability [14-18].

We performed a prospective randomized study to compare the efficacy of medial and lateral entry pinning 
Table 3 Base-line characteristics of 160 patients with displaced (Gartland [4] type II and III) extension type supracondylar fractures of humerus randomly assigned to receive percutaneous fixation with either crossed mediallateral pin or, two-lateral pins

\begin{tabular}{|c|c|c|c|}
\hline Baseline Characteristics & $\begin{array}{l}\text { Crossed medial-lateral pin entry group } \\
\qquad(\mathrm{n}=80)\end{array}$ & $\begin{array}{l}\text { Two-lateral pin entry group } \\
\qquad(\mathrm{n}=80)\end{array}$ & $\begin{array}{c}\mathrm{p} \\
\text { value }\end{array}$ \\
\hline Age + (years) & $6.24 \pm 1.77$ & $6.12 \pm 1.82$ & $0.67 \zeta$ \\
\hline Male sex $\uparrow(\%$ of patients): & $48(60)$ & $51(64)$ & $0.74 \psi$ \\
\hline Sideף(\% of patients): & & & $0.53 \psi$ \\
\hline Left & $68(85)$ & $64(80)$ & \\
\hline Right & $12(15)$ & $16(20)$ & \\
\hline Types of Displacement (\% of patients): & & & $0.71 \varnothing$ \\
\hline Posterolateral & $38(48)$ & $33(41)$ & \\
\hline Posteromedial & $24(30)$ & $28(35)$ & \\
\hline Posterior & $18(22)$ & $19(24)$ & \\
\hline $\begin{array}{l}\text { Types of fracture according to Gartland [4] (\% of } \\
\text { patients): }\end{array}$ & & & $0.75 \psi$ \\
\hline Type II & $34(42)$ & $37(46)$ & \\
\hline Type III & $46(58)$ & $43(54)$ & \\
\hline Interval from admission to surgery† (hours) & $25.4 \pm 10.26$ & $23 \pm 8.78$ & $0.11 \zeta$ \\
\hline Interval from injury to admissiont (hours) & $27.8 \pm 16.12$ & $29.47 \pm 11.74$ & $0.45 \zeta$ \\
\hline
\end{tabular}

† The datas are given as the mean \pm standard deviation. I The datas are given as the number (\%) of patients. $\zeta$ Independent-sample student $\mathrm{t}$ test. $\psi$ Fisher's exact. $\varnothing$ Chi-square test

with lateral entry pinning for percutaneous fixation of displaced (Gartland [4] type II and type III) extension type supracondylar fractures of the humerus in children.

In our study, we found no significant difference between the two groups with regard to iatrogenic ulnar nerve injury and loss of reduction grading.

Though seven studies $[11,14,18,23-26]$ have been done so far to compare the efficacy of medial and lateral entry pinning with lateral entry pinning for percutaneous fixation of displaced (Gartland [4] type II and type III) extension type supracondylar fractures of the humerus in children but, it is very difficult to compare between them because: (i) pinning technique, pin size, position of elbow during pinning differs in various studies, (ii) only one study [11] consists of more than 50 patients in each group but, that was a retrospective study, (iii) Most of the studies are retrospective and uncontrolled [11,14,18,24,25]. Only two studies $[23,26]$ are randomized controlled but, these studies consist of less than 50 patients in each group. All of these studies found no significant difference between the two methods in terms of loss of reduction and six studies found no significant difference between the two methods in terms of iatrogenic nerve injury. Only one shows significant difference in favor of lateral entry pinning method in terms of iatrogenic nerve injury. So, convincing evidence of the optimal method of percutaneous pin fixation is lacking in various literature overviews.

Brauer et al. [27] performed a systematic review using pooled data of 2054 children from 35 previous studies: 2 randomized trials, 6 retrospective studies and 25 case series. They found no significant difference between the two groups in terms of loss of reduction and iatrogenic nerve injury.

So, the results of our study are consistent with the results of most of the previous studies consists of the same clinically relevant question.

The major strength of the present study is its prospective randomized design. All of the patients in each group were operated on according to a uniform standardized well-accepted technique. Also, thorough follow-up

Table 4 Withdrawals of the 160 patients with displaced (Gartland [4] type II and III) extension type supracondylar fractures of humerus randomly assigned to receive percutaneous fixation with either crossed medial-lateral pin or, two-lateral pins

\begin{tabular}{llll}
\hline Lost to follow-up & Crossed medial-lateral pin entry group $(\mathbf{n}=\mathbf{8 0})$ & Two-lateral pin entry group $(\mathbf{n}=\mathbf{8 0})$ & $\mathbf{p}$ value \\
\hline After the surgery $\boldsymbol{\psi}$ & $2(3)$ & $2(3)$ & $1.0 \rho$ \\
\hline After the first visit $\boldsymbol{\psi}$ & $5(6)$ & $4(5)$ & $1.0 \rho$ \\
\hline After the second visit $\boldsymbol{\psi}$ & $9(11)$ & $8(10)$ & $1.0 \rho$ \\
\hline
\end{tabular}

$\psi$ The datas are given as the number (\%) of patients. $\rho$ Fisher's exact test 
Table 5 Complications of the 160 patients with displaced (Gartland [4] type II and III) extension type supracondylar fractures of humerus randomly assigned to receive percutaneous fixation with either crossed medial-lateral pin or, two-lateral pins

\begin{tabular}{|c|c|c|c|}
\hline Complications & $\begin{array}{l}\text { Crossed medial-lateral pin entry group }+ \\
(n=80)\end{array}$ & $\begin{array}{l}\text { Two-lateral pin entry group* } \\
(\mathrm{n}=80)\end{array}$ & $\begin{array}{l}\mathrm{p} \\
\text { value }\end{array}$ \\
\hline $\begin{array}{l}\text { Neurovascular complications at the time of } \\
\text { admission } \psi\end{array}$ & & & 0.759 \\
\hline Radial nerve injury & $6(8)$ & $5(6)$ & \\
\hline Median nerve injury & $9(11)$ & $12(15)$ & \\
\hline Pulseless pink hand & $7(9)$ & $6(8)$ & \\
\hline latrogenic ulnar nerve injury $\psi$ & 0 & 0 & $1.0 \rho$ \\
\hline $\begin{array}{l}\text { Pin track infection at three weeks follow-up visit } \\
\psi\end{array}$ & $2(3)$ & $3(4)$ & $1.0 \rho$ \\
\hline
\end{tabular}

assessment of each patient was done with the use of various clinical and radiological outcome measures at standardized intervals. Follow-up assessment of each patient was done by the same doctor throughout the trial.

The major limitation of our study is that, both the surgeon and the patients were not blinded of the treatment received throughout the trial. Another weakness of our study is the number of patients who did not complete the three-month follow-up visit. However, as the rate of the patients lost to follow-up in our study is comparable with that in other studies, we do not believe that it hampers our results.

In conclusion, we found that if a uniform standardized operative technique is followed in each method, then the result of both the percutaneous fixation methods will be same in terms of safety and efficacy.

Table 6 Comparative outcome measures at three months after the surgery in both groups

\begin{tabular}{|c|c|c|c|}
\hline Outcome measure & $\begin{array}{l}\text { Crossed medial-lateral pin entry group } \dagger \\
(n=64)\end{array}$ & $\begin{array}{l}\text { Two-lateral pin entry group * } \\
(n=66)\end{array}$ & $\begin{array}{l}\mathrm{p} \\
\text { Value }\end{array}$ \\
\hline Carrying angle (degree) $\pi$ & $5.52 \pm 3.77$ & $5.56 \pm 4.62$ & $0.95 \zeta$ \\
\hline Loss of Carrying angle (degree) $\pi$ & $3.58 \pm 3.08$ & $3.86 \pm 3.33$ & $0.62 \zeta$ \\
\hline \multicolumn{4}{|l|}{ Passive range of elbow motion (degree) $\pi$} \\
\hline Flexion & $128.3 \pm 12.67$ & $127.96 \pm 4.38$ & $0.75 \zeta$ \\
\hline Extension & $-2.6 \pm-0.13$ & $-2.56 \pm-0.16$ & $0.12 \zeta$ \\
\hline Total range of motion & $130.58 \pm 3.9$ & $129.39 \pm 4.48$ & $0.11 \zeta$ \\
\hline $\begin{array}{l}\text { Loss of total passive range of elbow motion } \\
\text { (degree) } \pi\end{array}$ & $3.4 \pm 2.9$ & $3.8 \pm 3.21$ & $0.45 \zeta$ \\
\hline Flynn grading (\% of patients) 9 & & & $0.84 \psi$ \\
\hline Excellent & $51(80)$ & $48(73)$ & \\
\hline Good & 6(9) & $8(12)$ & \\
\hline Fair & $7(11)$ & $10(15)$ & \\
\hline Poor & 0 & 0 & \\
\hline Loss of reduction grading (\% of patients) & & & $0.94 \psi$ \\
\hline Major & 0 & 0 & \\
\hline Mild & $9(15)$ & $8(12)$ & \\
\hline None & $55(85)$ & $58(88)$ & \\
\hline Baumann angle (degree) $\pi$ & $77.2 \pm 4.35$ & $76.2 \pm 3.51$ & $0.15 \zeta$ \\
\hline Change in the Baumann angle (degree) $\pi$ & $3.57 \pm 2.43$ & $3.71 \pm 2.1$ & $0.72 \zeta$ \\
\hline
\end{tabular}

† Data were missing for 16 patients * Data were missing for 14 patients

$\pi$ The datas are given as the mean \pm standard deviation

I The datas are given as the number (\%) of patients

$\zeta$ Independent-sample student $\mathrm{t}$ test

$\psi$ Chi-square test 


\section{Author details}

'Department of Orthopaedics, Burdwan Medical College, Burdwan, West Bengal, India. ${ }^{2}$ Department of Anaesthesiology, Burdwan Medical College, Burdwan, West Bengal, India. ${ }^{3} \mathrm{c} / \mathrm{o}$ Mr. Kalipada Maity, Bhatchala (Near Banka Bridge), PO Sripally Dist., Burdwan, West Bengal, India PIN-713103.

\section{Authors' contributions}

AM carried out the monitoring of data collection, patient follow-up, randomization etc. DS carried out the monitoring of operation under anaesthesia. DSR carried out the monitoring of surgical technique. All authors read and approved the final manuscript.

\section{Competing interests}

The authors declare that they have no competing interests.

Received: 20 June 2011 Accepted: 15 February 2012

Published: 15 February 2012

\section{References}

1. Otsuka NY, Kasser JR: Supracondylar fractures of the humerus in children. J Am Acad Orthop Surg 1997, 5:19-26.

2. Beaty JH, Kasser JR: Fractures about the elbow. Instr Course Lect 1995, 44:199-215.

3. Boyd HB, Altenberg AR: Fractures about the elbow in children. Arch Surg $1944,49: 213$.

4. Gartland JJ: Management of supracondylar fractures of the humerus in children. Surg Gynecol Obstet 1959, 109:145-154.

5. Mehlman CT, Crawford AH, McMillion TL, Roy DR: Operative treatment of supracondylar fractures of the humerus in children: the Cincinnati experience. Acta Orthop Belg 1996, 62(Suppl 1):41-50.

6. Flynn JC, Matthews JG, Benoit RL: Blind pinning of displaced supracondylar fractures of the humerus in children. Sixteen years' experience with long-term follow-up. J Bone Joint Surg Am 1974, 56:263-272.

7. Arino VL, Llurch EE, Ramriez AM, Ferrer J, Rodriguez L, Baixauli F: Percutaneous fixation of supracondylar fractures of the humerus in children. J Bone Joint Surg Am 1977, 59:914-916.

8. Chai KK: A Prospective study on supracondylar fractures of the humerus in children: comparing the results of closed manipulation and plaster cast with closed manipulation and percutaneous cross $\mathrm{K}$ wiring for the treatment of displaced fractures. Master Thesis: University of Malaya 2000, PMID:1 1200043.

9. Swenson AL: The treatment of supracondylar fractures of the humerus By Kirschner-wire transfixion. J Bone Joint Surg Am 1948, 30:993-997.

10. Casiano E: Reduction and fixation by pinning "banderillero" stylefractures of the humerus at the elbow in children. Mil Med 1960, 125:262-264

11. Skaggs DL, Hale JM, Bassett J, Kaminsky C, Kay RM, Tolo VT: Operative treatment of supracondylar fractures of the humerus in children. The consequences of pin placement. J Bone Joint Surg Am 2001, 83:735-740, PMID:11379744.

12. Lyons JP, Ashley E, Hoffer MM: Ulnar nerve palsies after percutaneous crosspinning of supracondylar fractures in children's elbows. J Pediatr Orthop 1998, 18:43-45, PMID:9449100.

13. Rasool MN: Ulnar nerve injury after K-wire fixation of supracondylar humerus fractures in children. J Pediatr Orthop 1998, 18:686-690, PMID:9746427.

14. Gordon JE, Patton CM, Luhmann SJ, Bassett GS, Schoenecker PL: Fracture stability after pinning of displaced supracondylar distal humerus fractures in children. J Pediatr Orthop 2001, 21:313-318, PMID: 11371812.

15. Lee SS, Mahar AT, Miesen D, Newton PO: Displaced pediatric supracondylar humerus fractures: biomechanical analysis of percutaneous pinning techniques. J Pediatr Orthop 2002, 22:440-443, PMID: 12131437.

16. Zionts LE, McKellop HA, Hathaway R: Torsional strength of pin configurations used to fix supracondylar fractures of the humerus in children. J Bone Joint Surg Am 1994, 76:253-256, PMID: 8113261.

17. Kallio PE, Foster BK, Paterson DC: Difficult supracondylar elbow fractures in children: analysis of percutaneous pinning technique. J Pediatr Orthop 1992, 12:11-15, PMID: 1732288.
18. Davis RT, Gorczyca JT, Pugh K: Supracondylar humerus fractures in children. Comparison of operative treatment methods. Clin Orthop Relat Res 2000, 376:49-55, PMID: 10906857.

19. Green DW, Widmann RF, Frank JS, Gardner MJ: Low incidence of ulnar nerve injury with crossed pin placement for pediatric supracondylar humerus fractures using a mini-open technique. J Orthop Trauma 2005, 19:158-163, PMID:15758668.

20. Aronson DD, Prager BI: Supracondylar fractures of the humerus in children: a modified technique for closed pinning. Clin Orthop Relat Res 1987, 219:174, PMID: 3581569.

21. Williamson DM, Coates CJ, Miller RK, Cole WG: Normal characteristics of the Baumann (humerocapitellar) angle: an aid in assessment of supracondylar fractures. J Pediatr Orthop 1992, 12:636-639, PMID: 1517426.

22. Skaggs DL, Cluck MW, Mostofi A, Flynn JM, Kay RM: Lateral-entry pin fixation in the management of supracondylar fractures in children. $J$ Bone Joint Surg Am 2004, 86:702-707, PMID: 15069133.

23. Foead A, Penafort R, Saw A, Sengupta S: Comparison of two methods of percutaneous pin fixation in displaced supracondylar fractures of the humerus in children. J Orthop Surg (Hong Kong) 2004, 12:76-82, PMID: 15237126.

24. Topping RE, Blanco JS, Davis TJ: Clinical evaluation of crossed-pin versus lateral-pin fixation in displaced supracondylar humerus fractures. J Pediatr Orthop 1995, 15:435-439, PMID:7560029.

25. Shamsuddin SA, Penafort R, Sharaf I: Crossed-pin versus lateral-pin fixation in pediatric supracondylar fractures. Med J Malaysia 2001, 56(Suppl D):38-44, PMID: 14569765.

26. Kocher MS, Kasser JR, Waters PM, Bae D, Snyder BD, Hresko MT, Hedequist D, Karlin L, Kim YJ, Murray MM, Millis MB, Emans JB, Dichtel L, Matheney T, Lee BM: Lateral entry compared with medial and lateral entry pin fixation for completely displaced supracondylar humeral fractures in children. A randomized clinical trial. J Bone Joint Surg Am 2007, 89:706-712.

27. Brauer CA, Lee BM, Bae DS, Waters PM, Kocher MS: A systematic review of medial and lateral entry pinning versus lateral entry pinning for supracondylar fractures of the humerus. J Pediatr Orthop 2007, 27:181-186, PMID: 17314643

\section{doi:10.1186/1749-799X-7-6}

Cite this article as: Maity et al: A prospective randomised, controlled clinical trial comparing medial and lateral entry pinning with lateral entry pinning for percutaneous fixation of displaced extension type supracondylar fractures of the humerus in children. Journal of Orthopaedic Surgery and Research 2012 7:6.

\section{Submit your next manuscript to BioMed Central and take full advantage of:}

- Convenient online submission

- Thorough peer review

- No space constraints or color figure charges

- Immediate publication on acceptance

- Inclusion in PubMed, CAS, Scopus and Google Scholar

- Research which is freely available for redistribution

Submit your manuscript at www.biomedcentral.com/submit
C Biomed Central 